\title{
TE Magnetostatic Surface Waves in Symmetric Dielectric Negative Permittivity Material Waveguide
}

\author{
A. I. Ass'ad' ${ }^{1}$ and H. S. Ashour ${ }^{2}$ \\ ${ }^{1}$ The Department of Physics, Al-Aqsa University, Gaza, Gaza Strip, Palestine \\ ${ }^{2}$ The Department of Physics, Al-Azhar University-Gaza, Gaza, Gaza Strip, Palestine
}

Correspondence should be addressed to H. S. Ashour, hashour@alazhar-gaza.edu

Received 18 December 2008; Revised 8 April 2009; Accepted 13 April 2009

Recommended by Leonid Pryadko

Nonlinear magnetostatic surface wave in a slab waveguide structure has been investigated. The design consisted of dielectric film between two thick nonlinear nonmagnetic negative permittivity material (NPM) layers. A dispersion relation for TE nonlinear Magnetostatic surface waves (NMSSWs) has been derived into the proposed structure and has been numerically investigated. Effective refractive index decreases with thickness and frequency increase have been found. Effective refractive index decrease with optical nonlinearity increase and switching to negative values of effective refractive index at a certain value of optical nonlinearity have been found. This meant that the structure behaved like a left-handed material over certain range. We found that the power flow was changing by changing the operating frequency, the dielectric film thickness, and the optical nonlinearity. Also, the effective refractive index and power flow attained constant values over certain values of dielectric constant values.

Copyright (C) 2009 A. I. Ass'ad and H. S. Ashour. This is an open access article distributed under the Creative Commons Attribution License, which permits unrestricted use, distribution, and reproduction in any medium, provided the original work is properly cited.

\section{Introduction}

Great interest is focused on the propagation of electromagnetic waves in artificial materials, and particularly on materials with negative index of refraction: materials which exhibited both negative permeability and permittivity over a certain range of frequencies. In those materials, there were the wave vector, the electric field, and the magnetic field form a left-handed system. Thus, they were called left-handed materials (LHMs).

A group of researchers at the University of San Diego was able to synthesize an artificial dielectric medium (metamaterials). They were able to demonstrate that those materials exhibit both negative dielectric permittivity and magnetic permeability simultaneously over a certain range of frequencies [1]. By that realization, the prediction of Veselago [2] in his pioneer paper that electromagnetic propagation in an isotropic medium with negative dielectric permittivity $\varepsilon(\omega)<0$ and negative permeability $\mu(\omega)<$ 0 that could exhibit unusual properties were realized. In such (LHMs) there appeared the electric field vector $E$, the magnetic field vector $H$, and the wave vector $k$ form a left- hand orthogonal set. Those recent demonstrations on the existence of the LHM resulted in a wide open to unique possibilities in the design of a novel type of device based on electromagnetic wave propagation in those materials, but in a nonconventional way.

Recently, Shadrivov et al. $[8,9]$ proposed a nonlinear LHM structure; Podolskiy and Narimanov [11] have proposed nonmagnetic linear LHM.

Recent studies by Assa'd et al. [6, 7] had proposed a new class of materials based on Shadrivov's and others work, which was the nonlinear nonmagnetic negative permittivity materials (NPMs). The nonlinear nonmagnetic NPM behaves like a metal with a better advantage; we can control its physical characteristics and the operating frequency range. This controllability is not widely available when use certain class of metal compared to NPMs [6].

Since most communication devices (e.g., waveguides and microstrips) include dielectric materials, which invited us to investigate a slab waveguide structure of dielectric film between two thick layers of nonlinear nonmagnetic negative permittivity material (NPM). 
The propagation of TE waves NMSSW in slab waveguide structure was investigated. They were dielectric film between two layers of nonlinear nonmagnetic negative permittivity material (NPM).

This paper has been organized as follows. Section 2 derived the dispersion relation of the surface waves in nonlinear nonmagnetic NPM dielectric nonlinear nonmagnetic NPM structure, and the power flow. In Section 3, we discussed the numerical results. Section 4 was solely devoted to the conclusion.

\section{Theory}

The dispersion relation for TE wave propagation in the $x$-axis with propagation wave constant $\beta$ is represented in the form $e^{i k_{0}(\beta x-c t)}$, where $\beta=k / k_{0}, k$ is the complex propagation constant, and $k_{0}$ is the free space wave number which equals $\omega / c$, where $c$ is the velocity of light, and $\omega$ is the applied angular frequency.

In Figure 1, the linear dielectric film of thickness $t$ and dielectric constant $\varepsilon$ is bounded by two layers of nonlinear nonmagnetic NPM in the regions $z<0$ and $z>t$, and its dielectric constant is $\varepsilon_{\text {eff }}^{\mathrm{NL}}$. Here we consider the Kerr-like nonlinearity of the dielectric in the composite material [810], that is,

$$
\varepsilon_{\mathrm{eff}}^{\mathrm{NL}}=\varepsilon_{D}\left(|E|^{2}\right)-\frac{\omega_{p}^{2}}{\omega^{2}},
$$

where $\omega_{p}$ is the plasma frequency, $\omega$ is the applied frequency, and $\varepsilon_{D}=\varepsilon_{1}+\alpha E_{y}^{2}, \varepsilon_{1}$ is the linear part of the dielectric constant, and $\alpha$ is the nonlinear coefficient. The permeability of the nonlinear nonmagnetic NPM is considered $\mu=1[6$, 11]. The electromagnetic field components are

$$
\begin{aligned}
E & =\left[0, E_{y}, 0\right] \exp \left(i k_{0}(\beta z-c t)\right), \\
H & =\left[H_{x}, 0, H_{z}\right] \exp \left(i k_{0}(\beta z-c t)\right) .
\end{aligned}
$$

2.1. In Nonlinear Nonmagnetic NPM $(z>t$ and $z<$ t). Substitution of (2) into Maxwell's equation yields the following nonlinear differential equation to satisfy in the nonlinear nonmagnetic NPM:

$$
\frac{\partial^{2} E_{y}}{\partial z^{2}}-k_{0}^{2}\left(\beta^{2}-\varepsilon_{D D}-\alpha E_{y}^{2}\right) E_{y}=0,
$$

where, $\varepsilon_{D D}=\varepsilon_{1}-\left(\omega_{p}^{2} / \omega^{2}\right)$.

The solution of equation (3) is given by [12].

$$
\begin{aligned}
& E_{y 1}=\left(\frac{k_{1}}{k_{0}}\right)\left(\frac{2}{\alpha}\right)^{1 / 2} \operatorname{sech}\left(k_{1}\left(z-z_{0}\right)\right), \quad z>t, \\
& E_{y 3}=\left(\frac{k_{1}}{k_{0}}\right)\left(\frac{2}{\alpha}\right)^{1 / 2} \operatorname{sech}\left(k_{3}\left(z-z_{0}\right)\right), \quad z<t,
\end{aligned}
$$

where $z_{0}$ is the position of the maximum of the field component in the nonlinear cover, and $k_{1}=k_{0} \sqrt{\beta^{2}-\varepsilon_{D D}}$.

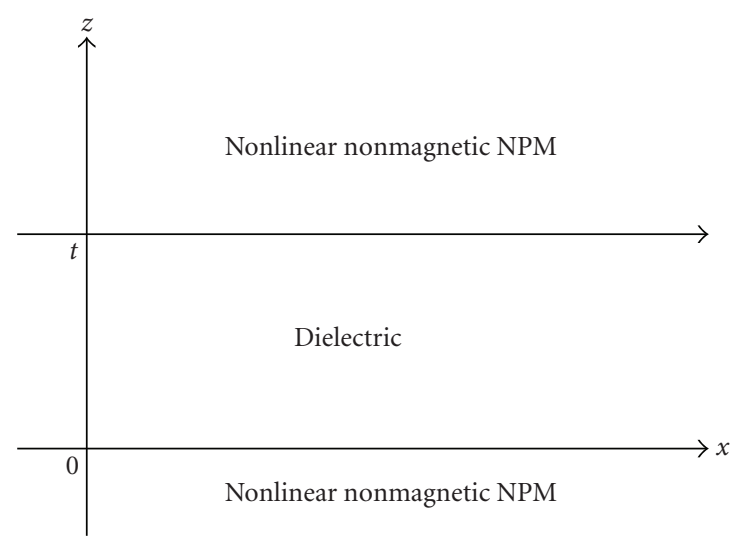

FIGURE 1: symmetric waveguide structure; linear dielectric medium nonlinear-bounded by nonlinear nonmagnetic NPM layers.

The magnetic field components in the nonlinear cover at $z>t$ are

$$
H_{x 1}=i\left(\frac{k_{1}^{2}}{\omega \mu_{0} k_{0}}\right)\left(\frac{2}{\alpha}\right)^{1 / 2} \operatorname{sech}\left(k_{1}\left(z-z_{0}\right)\right) \tanh \left(k_{1}\left(z-z_{0}\right)\right),
$$

$$
H_{z 1}=-\left(\frac{k}{\omega \mu_{0}}\right) E_{y 1}
$$

and at $z<t$

$$
\begin{aligned}
& H_{x 3}=i\left(\frac{k_{3}^{2}}{\omega \mu_{0} k_{0}}\right)\left(\frac{2}{\alpha}\right)^{1 / 2} \operatorname{sech}\left(k_{3}\left(z-z_{0}\right)\right) \tanh \left(k_{3}\left(z-z_{0}\right)\right), \\
& H_{z 3}=-\left(\frac{k}{\omega \mu_{0}}\right) E_{y 3} .
\end{aligned}
$$

2.2. In Linear Dielectric Region $(0<z<t)$. Also, substitution of (2) into Maxwell's equation yields the following linear differential equation to satisfy in the linear dielectric film:

$$
\frac{\partial^{2} E_{y}}{\partial z^{2}}-k_{0}^{2}\left(\beta^{2}-\varepsilon_{2}\right) E_{y}=0
$$

The solution of (6) is given by

$$
E_{y 2}=A \sinh \left(k_{2} z\right)+B \cosh \left(k_{2} z\right),
$$

where $k_{2}=k_{0} \sqrt{\beta^{2}-\varepsilon_{2}}$. The magnetic field components in the linear dielectric are

$$
\begin{aligned}
& H_{x 2}=-\left(\frac{i}{\omega \mu_{0}}\right) k_{2}\left[A \cosh \left(k_{2} z\right)+B \sinh \left(k_{2} z\right)\right], \\
& H_{z 2}=-\left(\frac{k}{\omega \mu_{0}}\right)\left[A \sinh \left(k_{2} z\right)+B \cosh \left(k_{2} z\right)\right] .
\end{aligned}
$$




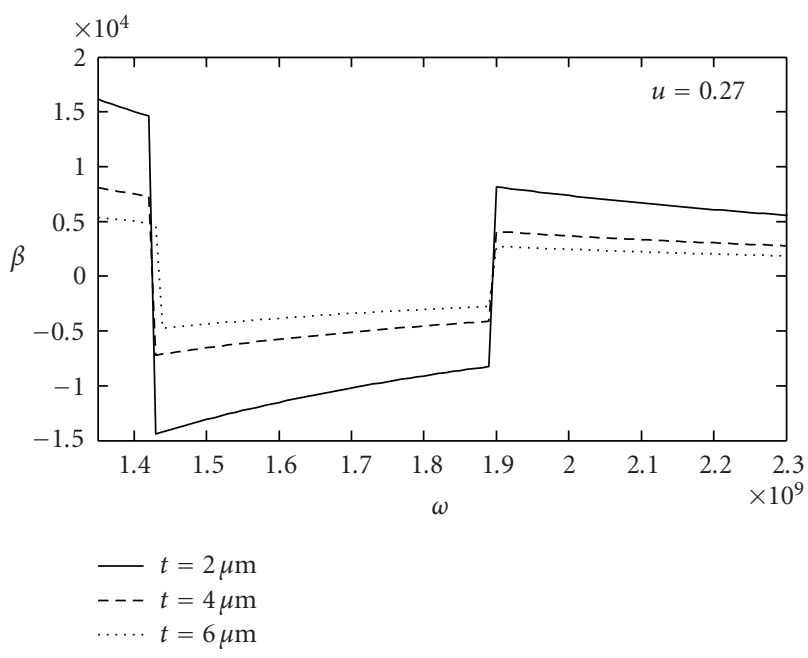

FIGURE 2: The effective nonlinear refractive index versus the angular frequency at nonlinearity $u=0.27$, for different dielectric slab thicknesses.

The dispersion relation can be found by matching the field components at the interface $z=0$ and $z=t$, that is,

$$
\left(\frac{k_{2}}{k_{1}}\right) v=\frac{R \tanh \left(k_{2} t\right)+1}{R+\tanh \left(k_{2} t\right)},
$$

where $R=\left(k_{1} / k_{2}\right) u, v=\left(u-\tanh \left(k_{1} t\right)\right) /\left(1+\tanh \left(k_{1} t\right) u\right)$, and $u=\tanh \left(k_{1} z_{0}\right)$ is the optical nonliearaty.

Power Flow. The power flux of the TE surface waves along the direction of propagation can be found by integrating the Poynting vector

$$
\begin{aligned}
P & =\frac{1}{2} \int\left(E \times H^{*}\right) d z=\frac{1}{2} \int E_{y} H_{z} d z=P_{1 \mathrm{NL}}+P_{D}+P_{2 \mathrm{NL}}, \\
P_{\mathrm{NL} 1} & =\frac{1}{2}\left(\frac{k k_{1}}{k_{0}^{2} \omega \mu_{0}}\right)\left(\frac{2}{\alpha_{1}}\right)(u+1), \\
P_{D} & =\frac{1}{2}\left(\frac{k \mu o}{\omega}\right) B^{2}\left[P_{1 D}+P_{2 D}+P_{3 D}\right],
\end{aligned}
$$

where

$$
\begin{aligned}
B & =\frac{k_{1}}{k_{0}}\left(\frac{2}{\alpha_{1}}\right)^{1 / 2}\left(1-v^{2}\right)^{1 / 2}\left[R \sinh \left(k_{2} t\right)+\cosh \left(k_{2} t\right)\right]^{-1}, \\
P_{1 D} & =\frac{R^{2}\left(\cosh \left(k_{2} t\right) \sinh \left(k_{2} t\right)-k_{2} t\right)}{2 k_{2}}, \\
P_{2 D} & =\frac{\cosh \left(k_{2} t\right) \sinh \left(k_{2} t\right)+k_{2} t}{2 k_{2}}, \\
P_{3 D} & =\frac{R \sinh ^{2}\left(k_{2} t\right)}{k_{2}} \\
P_{\mathrm{NL} 2} & =\frac{1}{2}\left(\frac{k k_{1}}{k_{0}^{2} \omega \mu_{0}}\right)\left(\frac{2}{\alpha_{1}}\right)[1+\nu] .
\end{aligned}
$$

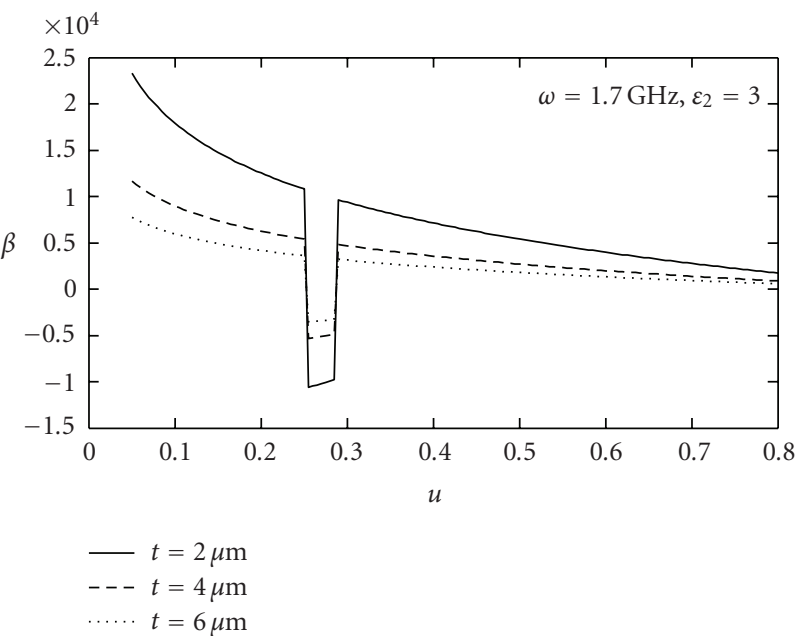

FIGURE 3: The effective nonlinear refractive index versus the nonlinearity, at $\omega=1.7 \mathrm{GHz}, \varepsilon_{2}=3$, for different dielectric film thicknesses.

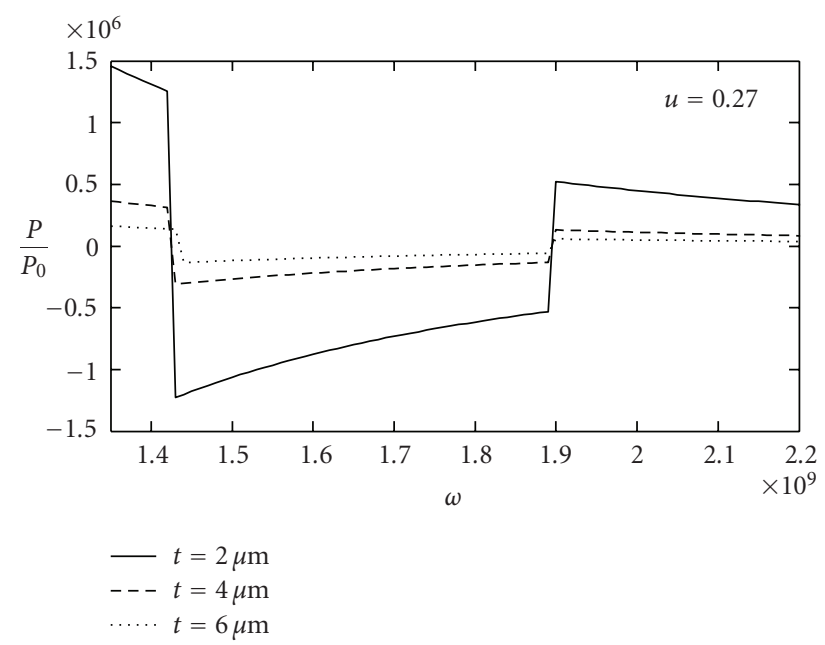

FIGURE 4: The normalized power flux versus the operating angular frequency at nonlinearity $u=0.27$, for different dielectric film thicknesses.

\section{Numerical Results and Discussion}

The dispersion relation, (11), numerically solved to find the complex effective wave index $\beta$ as a function of the angular frequency $\omega$, and the optical nonlinearity $u$ for different values of dielectric film thicknesses $(t=2,4$, and $6 \mu \mathrm{m})$. The power flux for the structure under investigation has been investigated for the film thicknesses. Besides that, we investigated the effect of the dielectric constant of the dielectric material. The parameters of the nonlinear nonmagnetic negative permittivity NPM and linear dielectric are adjusted so that the parameter $\varepsilon_{\text {eff }}^{\mathrm{NL}}$ is negative in the same frequency range, which lies between $1.3 \sim 2.2 \mathrm{GHz}$. The parameters that were used in carrying out the numerical calculations are $[6,13]: \omega_{p}=2 \pi \mathrm{GHz}, \alpha=1.55 \times 10^{-10} \mathrm{~m}^{2} \mathrm{~V}^{-2}, \varepsilon_{1}=$ $2.5, \varepsilon_{2}=3.0$, and $\omega_{0}=10.21 \mathrm{GHz}$. 


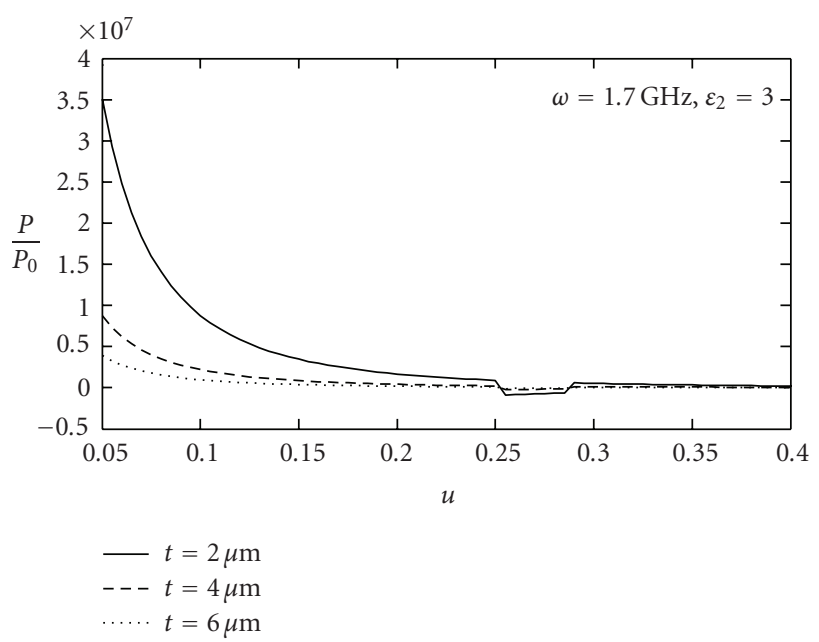

(a)

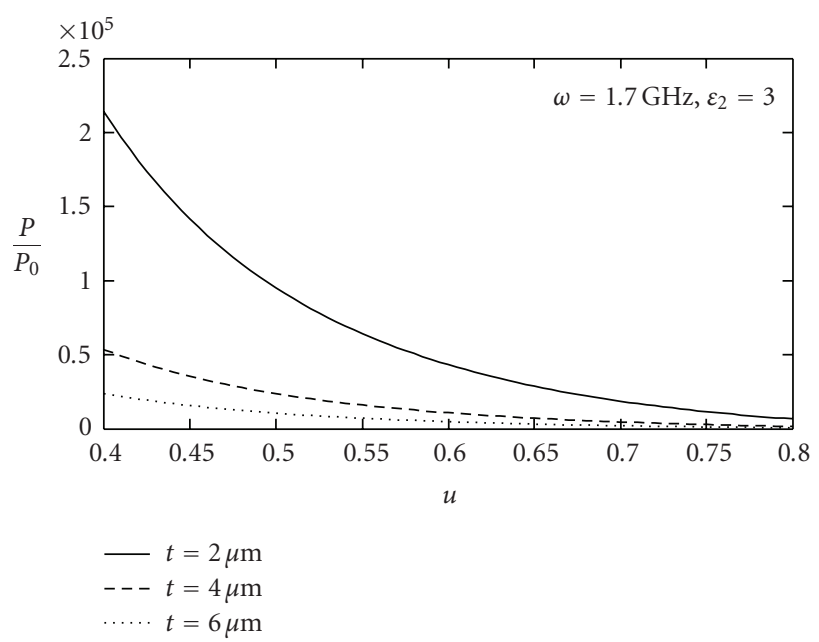

(b)

Figure 5: The normalized power flux versus optical nonlinearity at operating angular frequency and dielectric constant, $\omega=1.7 \mathrm{GHz}, \varepsilon_{2}=$ 3 , for different dielectric film thicknesses.

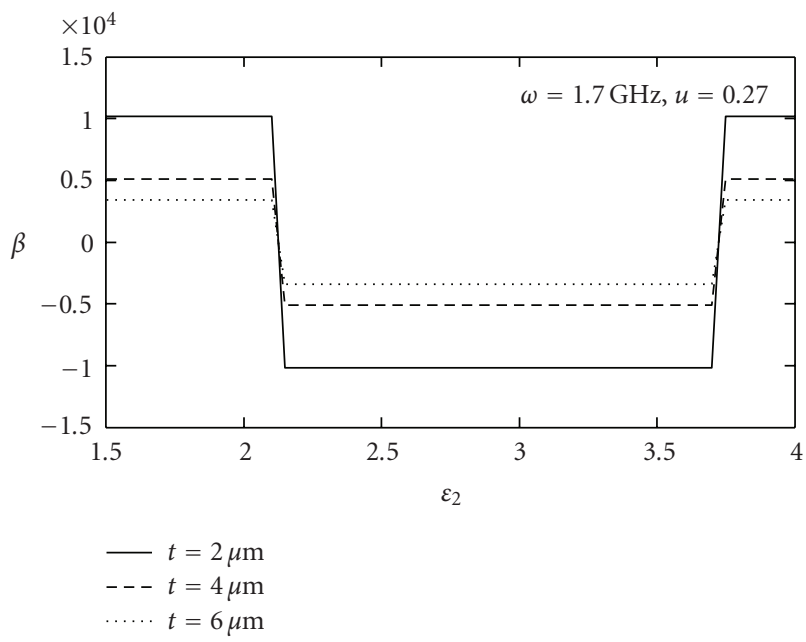

Figure 6: The effective refractive index versus dielectric constant at operating angular frequency and optical nonlinearity, $\omega=$ $1.7 \mathrm{GHz}, u=0.27$, for different dielectric film thicknesses.

In Figure 2, we plotted the effective refractive index, $\beta$, versus the frequency range at optical nonlinearity, $u=$ 0.27 , at different dielectric film thicknesses. It is noticed that the effective refractive index is smoothly decreasing with frequency increase and negative slope. In the middle range of frequencies the effective refractive index switches to negative values with positive slope. After that, at higher frequencies the effective refractive index switches back to positive values with negative slope again. Thus, the structure behaved like left-handed material (LHM) in the middle range frequencies. The slope of the dispersion relation represents the group velocity. When the slope has a negative gradient this means that the group velocity is negative, and the structure behaves like LHM. These properties are due to the fact that the Poynting vector in the nonlinear nonmagnetic

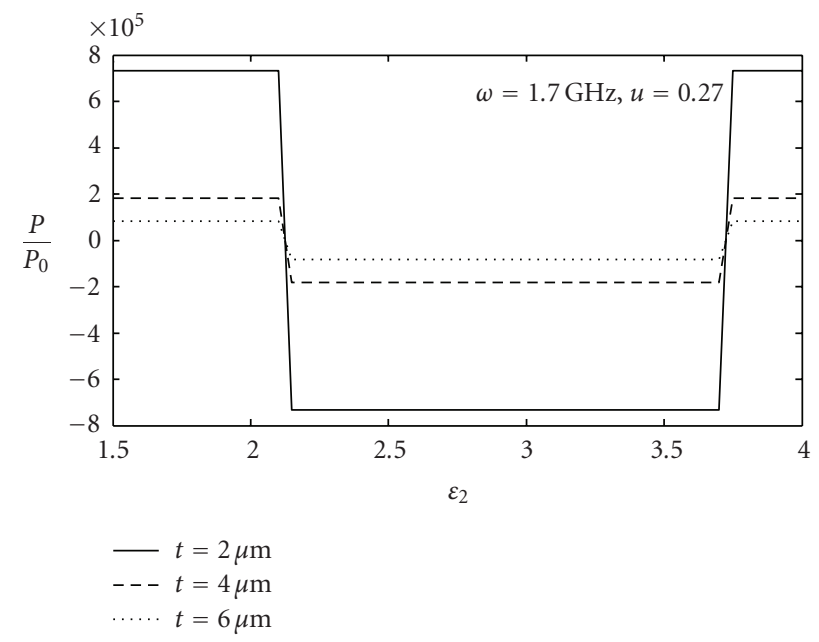

Figure 7: The normalized power flux versus dielectric constant at operating angular frequency and optical nonlinearity, $\omega=$ $1.7 \mathrm{GHz}, \quad u=0.27$, for different dielectric film thicknesses.

NPM is antiparallel to the wave number, which characterized by negative dielectric constant $[14,15]$.

The effective refractive index $\beta$ of the structure versus the nonlinearity $u$ at different film thickness and at angular frequency $\omega=1.7 \mathrm{GHz}$ is shown in Figure 3. We notice that the effective refractive index, $\beta$, of the structure smoothly decreases with the nonlinearity $u$, despite the small region in the nonlinearity $(u=0.25 \sim 0.28)$ in which drastic change happens in the values of the effective refractive index, it turns to negative values. We noticed that the effective refractive index is sensitive to the dielectric film thickness. The effective refractive index decreases with dielectric film thickness increase for all nonlinearity values.

Figure 4 shows the normalized power flow $\left(P / P_{0}\right)$, where $P_{0}=1 /\left(2 \alpha \varepsilon_{0} \omega\right)$, versus the operating frequency with optical 
nonlinearity $u=0.27$. At low frequencies in the range the power flow decreases with frequency increase. Then the power flow switches to negative values in the middle range frequencies. At high frequencies in the range the power flow switches to positive values. The power flow is sensitive to dielectric medium slab thickness. The power flow decreases with dielectric film thickness increase.

In Figures 5(a) and 5(b) (for clarity), we plotted the power versus the optical nonlinearity at $\omega=$ 1.7 GHz operating frequency and dielectric constant, $\varepsilon_{2}=$ 3. The power flow decreases with optical nonlinearity increase for all dielectric film thicknesses.

In Figures 6 and 7, we explored the effect of the dielectric constant on the effective refractive index of the structure and power flow. For some operating frequency range the effective refractive index of the structure and power flow have almost constant values. The effective refractive index and power flow decreases with dielectric film thickness increase for all dielectric constant values taken in the range.

\section{Conclusion}

We analytically studied the TE surface waves in a slab waveguide. It contained dielectric film between two nonlinear nonmagnetic negative permittivity material (NPM) thick layers. A dispersion relation for TE surface waves has been derived and numerically investigated. There found out that the wave effective refractive index was changing in value and sign depending on the operating frequency and the dielectric slab thickness. The effective refractive index and power flow were sensitive to optical nonlinearity and dielectric slab thicknesses; they decreased with nonlinearity and thickness increase. We noticed that the effective refractive index and power flow attained constant values, at certain values of nonlinearity and operating frequency, versus dielectric slab dielectric constant.

\section{References}

[1] R. A. Shelby, D. R. Smith, and S. Schultz, "Experimental verification of a negative index of refraction," Science, vol. 292, no. 5514, pp. 77-79, 2001.

[2] V. G. Veselago, "The electrodynamics of substances with simultaneously negative values of $\varepsilon$ and $\mu$," Soviet Physics Uspekhi, vol. 10, pp. 509-514, 1968, Uspekhi Fizicheskikh Nauk, vol. 92, pp. 517-526, 1967.

[3] J. B. Pendry, "Negative refraction makes a perfect lens," Physical Review Letters, vol. 85, no. 18, pp. 3966-3969, 2000.

[4] P. M. Valanju, R. M. Walser, and A. P. Valanju, "Wave refraction in negative-index media: always positive and very inhomogeneous," Physical Review Letters, vol. 88, no. 18, Article ID 187401, 4 pages, 2002.

[5] Z. Ye, "About negative refraction and left handed materials," Physical Review B, vol. 67, Article ID 193106, 2003.

[6] A. I. Ass'ad, H. S. Ashour, and M. M. Shabat, "Magnetostatic surface waves in ferrite-nonlinear nonmagnetic negative permittivity material structure," International Journal of Modern Physics B, vol. 21, no. 12, pp. 1951-1960, 2007.

[7] A. I. Ass'ad, H. S. Ashour, and M. M. Shabat, "Nonlinear TE surface waves in linear-nonlinear nonmagnetic LHM structure," Journal of Al-Azhar University, vol. 8, no. 2, pp. 7584,2006

[8] I. V. Shadrivov, A. A. Sukhorukov, and Y. S. Kivshar, "Nonlinear surface waves in left-handed materials," Physical Review E, vol. 67, Article ID 057602, 2003.

[9] I. V. Shadrivov, A. A. Sukhorukov, Y. S. Kivshar, A. A. Zharov, A. D. Boardman, and P. Egan, "Nonlinear surface waves in left-handed materials," Physical Review E, vol. 69, Article ID $016617,2004$.

[10] I. V. Shadrivov and Y. S. Kivshar, "Spatial solitons in nonlinear left-handed metamaterials," Journal of Optics A, vol. 7, no. 2, pp. S68-S72, 2005.

[11] V. A. Podolskiy and E. E. Narimanov, "Non-magnetic lefthanded material," Physical Review B, vol. 71, Article ID 201101, 2005.

[12] A. D. Boardman, M. M. Shabat, and R. F. Wallis, "TE waves at an interface between linear gyromagnetic and nonlinear dielectric media," Journal of Physics D, vol. 24, no. 10, pp. 1702-1707, 1991.

[13] A. I. Ass'ad, "Nonlinear TE surface waves in dielectric material sandwiched between LHM and nonlinear nonmagnetic LHM structure," Journal of Al-Aqsa University, vol. 10, 2005.

[14] M. S. Hamada, A. H. El-Astal, and M. M. Shabat, "Nonlinear surface waves at a single interface of semimagnetic semiconductor-left handed materials (LHM)," International Journal of Microwave and Optical Technology, vol. 2, no. 2, pp. 112-118, 2007.

[15] D. Mihalache, R. G. Nazmitdinove, and V. K. Fedyanin, "Nonlinear optical waves in layered structure," Soviet Journal of Particles and Nuclei, vol. 20, pp. 86-107, 1989. 

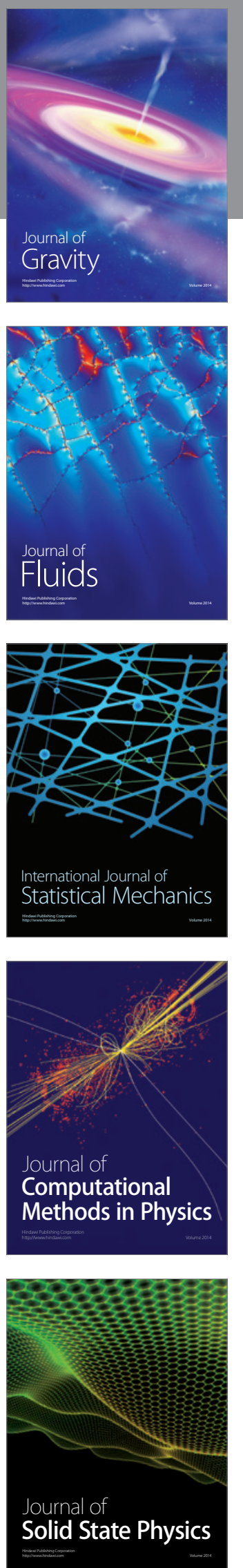

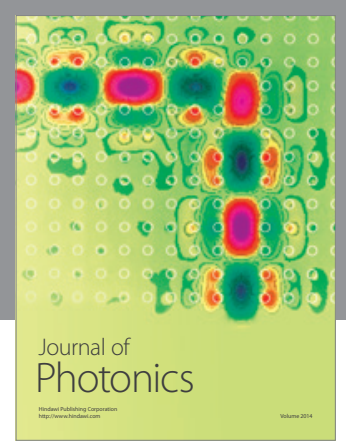

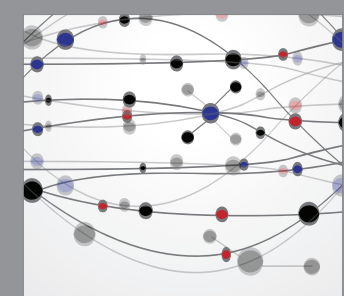

The Scientific World Journal
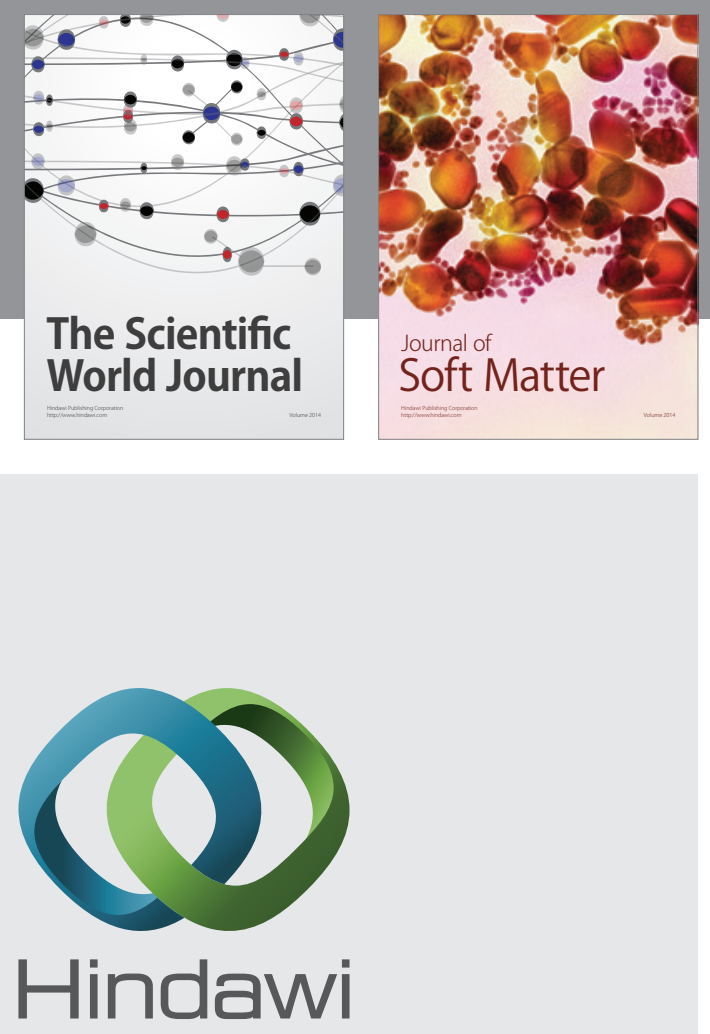

Submit your manuscripts at

http://www.hindawi.com
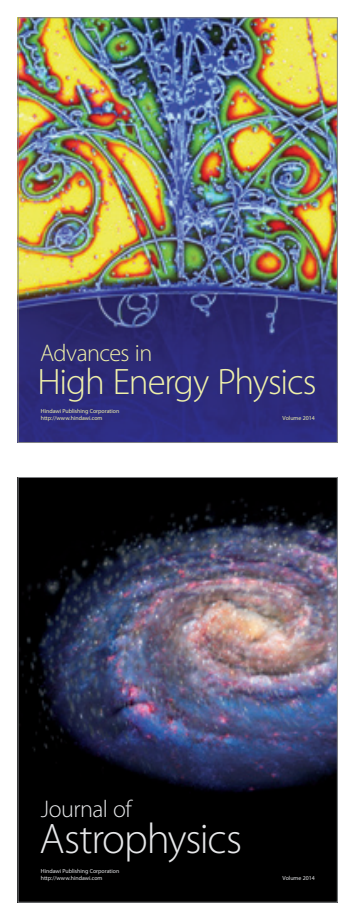
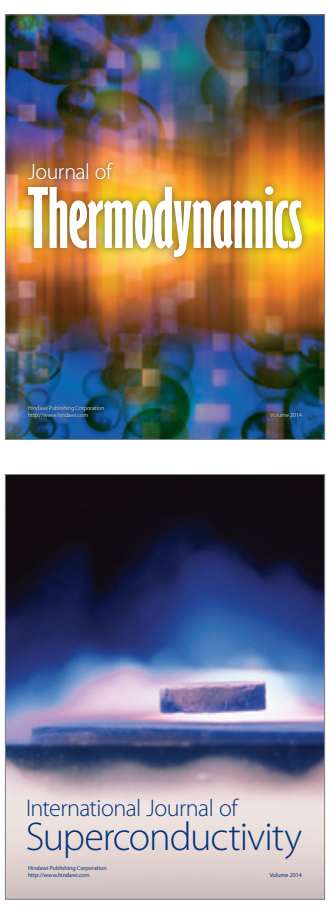
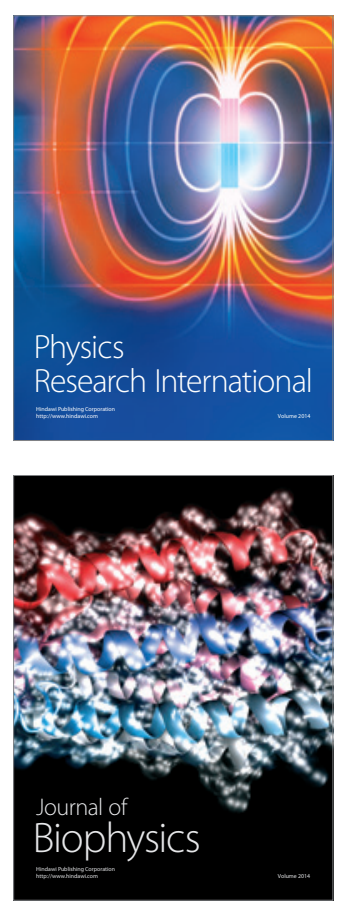
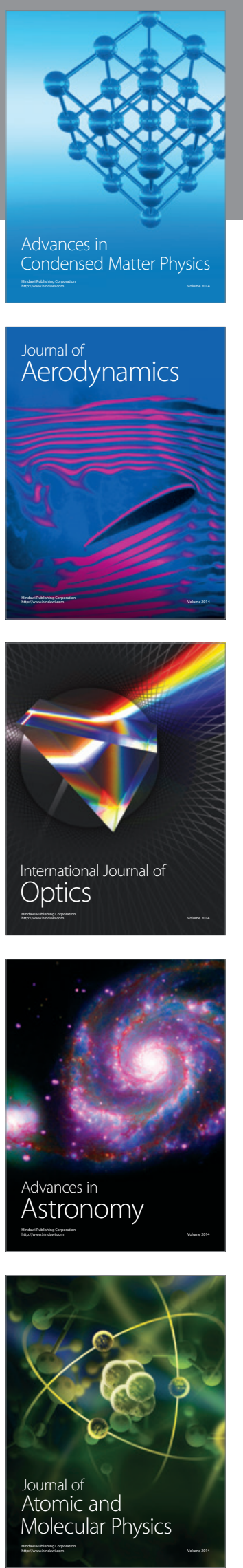